\title{
ON NORMAL DIVISION ALGEBRAS OF TYPE $R$ IN THIRTY-SIX UNITS*
}

\author{
BY \\ A. ADRIAN ALBERT
}

1. Introduction. A normal division algebra in $n^{2}$ units over a nonmodular field $F$ is of type $R$ if it contains a quantity $i$ whose minimum equation with respect to $F, \phi(\omega)=0$, has degree $n$ and $n$ distinct roots which are polynomials in $i$ with coefficients in $F$. Algebras of type $R$ occupy a central position in the theory of division algebras as they are the only normal division algebras whose structure is known, and all division algebras of order less than twenty-five are expressible as algebras of type $R$.

The normal division algebras $D$ whose structure is the simplest are those for the case where $\phi(\omega)=0$ has the cyclic group with respect to $F$. When $n$ is six and $\phi(\omega)=0$ is cyclic, $D$ is expressible as the direct product of a generalized quaternion division algebra and a cyclic division algebra of order nine, while conversely every such direct product is a cyclic division algebra of order thirty-six. The group of $\phi(\omega)=0$ is evidently regular and hence the only other type of equation to be considered for algebras of order thirty-six and type $R$ is one which has the single non-cyclic, non-abelian regular group on six letters, a case giving a very complicated algebra.

It has never been demonstrated that there exist normal division algebras which are not cyclic algebras. The author showed, in a recent paper, $\dagger$ that the algebras which had been constructed by $F$. Cecionif and which were based on a non-cyclic quartic were cyclic algebras. We show here that all normal division algebras of type $R$ in thirty-six units are cyclic algebras.

2. Algebras based on a non-cyclic sextic with regular group. Let $D$ be an associative normal division algebra of order thirty-six and type $R$, and let $i$ be the quantity of $D$ which defines the type of $D$. If $\phi(\omega)=0$, the minimum equation of $i$, is a cyclic sextic, $D$ is called a cyclic algebra. There remains to be considered the case where the group of $\phi(\omega)=0$ is non-cyclic. The author has shown $\|$ that $\phi(\omega)$ may be taken to have only even powers of the indeterminate $\omega$ and that there exists a polynomial $\theta(i)$ in $F(i)$ such that

* Presented to the Society, October 25, 1930; received by the editors in August, 1930.

† These Transactions, vol. 32 (1930), pp. 171-195.

$\ddagger$ Rendiconti del Circolo Matematico di Palermo, vol. 47 (1923), pp. 209-254.

|| See Theorem 12 of the author's paper, American Journal of Mathematics, vol. 52 (1930), pp. 283-292. 
(1) $\phi(\omega) \equiv\left[\omega+\theta^{2}(i)\right]\left[\omega-\theta^{2}(i)\right][\omega+\theta(i)][\omega-\theta(i)](\omega+i)(\omega-i)$, while for the non-cyclic case

$$
\theta^{3}(i)=i, \theta(-i)=-\theta^{2}(i), \theta^{2}(-i)=-\theta(i) .
$$

Evidently $i^{2}$ satisfies a cubic equation irreducible in $F$, and $F\left(i^{2}\right)$ is a cubic field over $F$. The set of all quantities in $F(i)$ which are symmetric in $i, \theta(i)$, $\theta^{2}(i)$, form a quadratic sub-field

$$
K=F(v), v^{2}=\rho \text { in } F,
$$

of $F(i)$. A cubic field contains no quadratic sub-field so $v$ is not in $F\left(i^{2}\right)$. Hence $1, v$ are linearly independent with respect to $F\left(i^{2}\right)$, and every quantity in $F(i)$ is expressible in the form

$$
a=a(i)=a_{1}+a_{2} v \quad\left(a_{1} \text { and } a_{2} \text { in } F\left(i^{2}\right)\right) .
$$

But then

$$
i=p_{1}+p v
$$

with $p$ and $p_{1}$ in $F\left(i^{2}\right)$, so that

$$
i^{2}=\left(p_{1}^{2}+p^{2} \rho\right)+2 p_{1} p v, 0=\left(p_{1}^{2}+p^{2} \rho-i^{2}\right)+2 p_{1} p v .
$$

It follows that $2 p_{1} p=0$. If $p$ were zero then $i$ would be in $F\left(i^{2}\right)$, a cubic field, contrary to the fact that $F(i)$ is a field of order six. Hence $p_{1}$ is zero and

$$
i=p v, p \text { in } F\left(i^{2}\right) \text {. }
$$

It is known* that $D$ has a basis

$$
i^{s} j^{t}, i^{s} j^{t} z \quad(s=0,1, \cdots, 5 ; t=0,1,2),
$$

and a multiplication table

$$
\begin{aligned}
\phi(i) & =0, & j^{t} a & =a\left[\theta^{t}(i)\right] j^{t} \quad(t=0,1, \cdots), \\
z a(i) & =a(-i) z, & z j & =\alpha j^{2} z, z j^{2}=\alpha \alpha\left[\theta^{2}(i)\right] g j z, \\
j^{3} & =g, & z^{2} & =\gamma,
\end{aligned}
$$

for every $a$ in $F(i)$ where $g, \alpha, \gamma$ are in $F(i)$ Since $z z^{2}=z^{2} z$ we have $\gamma=\gamma(-i)$ is in $F\left(i^{2}\right)$. Similarly $j g=g j$ gives $g=g[\theta(i)]$ is in $F(v)$. Write

$$
\gamma=\gamma_{1}+\gamma_{2} i^{2}+\gamma_{3} i^{4} \quad\left(\gamma_{1}, \gamma_{2}, \gamma_{3} \text { in } F\right),
$$

and suppose that $\gamma_{3} \neq 0$. We can then define scalars $\gamma_{5}, \gamma_{6}$ in $F$ by $\gamma_{1}=\gamma_{3} \gamma_{5}$, $\gamma_{2}=2 \gamma_{3} \gamma_{6}$ and

* See L. E. Dickson, Algebren und ihre Zahlentheorie, pp. 75-79, where $q=2, z \equiv j_{\Downarrow}, j \equiv j_{1}$, $\theta_{q}(i) \equiv-i, \theta_{1}(i) \equiv \theta(i)$. 


$$
\gamma=\gamma_{3}\left(i^{4}+2 \gamma_{6} i^{2}+\gamma_{6}^{2}+\gamma_{5}-\gamma_{6}^{2}\right)=\gamma_{3}\left(i^{2}+\gamma_{6}\right)^{2}+\gamma_{3}\left(\gamma_{5}-\gamma_{6}^{2}\right) .
$$

Consider the quantity

$$
i_{1}=\left(i^{2}+\gamma_{6}\right) v \text {. }
$$

Since $v \neq 0$ we have $v^{2}=\rho \neq 0$ in a division algebra and

$$
i_{1}^{2}=\left(i^{2}+\gamma_{6}\right)^{2} \rho=\rho i^{4}+2 \rho i^{2} \gamma_{6}+\rho \gamma_{6}^{2}
$$

is in $F\left(i^{2}\right)$ but not in $F$, since in particular the coefficient of $i^{4}$ is not zero. But $F\left(i^{2}\right)$ has no proper sub-field other than $F$, so that $F\left(i_{1}^{2}\right)=F\left(i^{2}\right)$. The quantities

$$
\begin{aligned}
\left(i^{2}+\gamma_{6}\right) v,-\left(i^{2}+\gamma_{6}\right) v, & {\left[\theta(i)^{2}+\gamma_{6}\right] v, } \\
- & {\left[\theta^{2}(i)^{2}+\gamma_{6}\right] v,\left[\theta^{2}(i)^{2}+\gamma_{6}\right] v,\left[\theta(i)^{2}+\gamma_{6}\right] v }
\end{aligned}
$$

are transforms

$$
i_{1}, \quad z i_{1} z^{-1}, \quad j i_{1} j^{-1}, \quad z j i_{1}(z j)^{-1}, \quad j^{2} i_{1} j^{-2}, \quad z j^{2} i_{1}\left(z j^{2}\right)^{-1}
$$

of $i_{1}$ and are roots of its minimum equation. If they were not distinct, two of

$$
\pm\left(i^{2}+\gamma_{6}\right), \pm\left[\theta(i)^{2}+\gamma_{6}\right], \pm\left[\theta^{2}(i)+\gamma_{6}\right]
$$

would be equal, which is impossible since those with plus signs are the distinct roots of the irreducible cubic minimum equation of $i^{2}+\gamma_{6}$, while this cubic has not the negative of any one of its roots as a root since it has not even powers only. The minimum equation of $i_{1}$ has thus six distinct roots in $F(i)$ so that its degree is $\operatorname{six}, F\left(i_{1}\right)$ contained in $F(i)$ has order six, and $F\left(i_{1}\right)=F(i)$. Evidently $z i_{1}=-i_{1} z$, while $j$ transforms $i_{1}$ into a quantity in $F\left(i_{1}\right)$, that is a polynomial in $i_{1}$. We may thus replace $i$ by $i_{1}$ in the basis of $D$ without loss of generality, and, since $\gamma=\left(\gamma_{3} \rho^{-1}\right) \vdots_{1}{ }^{2}+\gamma_{3}\left(\gamma_{5}-\gamma_{6}{ }^{2}\right)$, for this new $i$ we have $\gamma$ expressed as a linear combination with coefficients in $F$ of 1 and $i^{2}$. When $\gamma_{3}=0$ we also have immediately such an expression, so that we have proved

Lемма 1. The quantity $i$ may be so chosen that, without altering any other property of $D$,

$$
\gamma=\gamma_{1}+\gamma_{2} i^{2} \quad\left(\gamma_{1} \text { and } \gamma_{2} \text { in } F\right) .
$$

We shall utilize the notations

$$
a^{\prime}=a(-i), a_{\theta}=a[\theta(i)], a_{\theta \theta}=\left(a_{\theta}\right)_{\theta},
$$

so that from (2) we immediately have

$$
\begin{array}{ll}
\left(a^{\prime}\right)^{\prime}=a, & \left(a_{\theta}\right)_{\theta \theta}=\left(a_{\theta \theta}\right)_{\theta}=a, \\
\left(a^{\prime}\right)_{\theta}=\left(a_{\theta \theta}\right)^{\prime}, & \left(a^{\prime}\right)_{\theta \theta}=\left(a_{\theta}\right)^{\prime},
\end{array}
$$


for every $a$ of $F(i)$. Also

$$
j a=a_{\theta} j, j^{2} a=a_{\theta \theta} j^{2}, z a=a^{\prime} z,
$$

from (7), while

(13) $i^{\prime}=-i, v^{\prime}=-v, v_{\theta}=v,\left(i^{2}\right)^{\prime}=i^{2}, g=g_{\theta}=g_{\theta \theta}, \gamma=\gamma^{\prime}$.

Consider the quantities

$$
d=\lambda_{1}+\lambda_{4} i, e=\lambda_{2}+\lambda_{3} i
$$

where

$$
2 \lambda_{1}=1+\gamma_{1}, 2 \lambda_{2}=1-\gamma_{1}, 2 \lambda_{3}=1+\gamma_{2}, 2 \lambda_{4}=1-\gamma_{2},
$$

so that $\lambda_{1}, \cdots, \lambda_{4}$ are in $F, \lambda_{1}{ }^{2}-\lambda_{2}{ }^{2}=\gamma_{1}, \lambda_{3}{ }^{2}-\lambda_{4}{ }^{2}=\gamma_{2}$. Then

$$
d d^{\prime}-e e^{\prime}=\lambda_{1}{ }^{2}-\lambda_{4}{ }^{2} i^{2}-\left(\lambda_{2}{ }^{2}-\lambda_{3}{ }^{2} i^{2}\right)=\gamma_{1}+\gamma_{2} i^{2}=\gamma .
$$

But $\gamma=\gamma^{\prime}$ and if we put $f=d \gamma^{-1}, h=e \gamma^{-1}$, we have

$$
\left(f f^{\prime}-h h^{\prime}\right) \gamma=\left(d d^{\prime}-e e^{\prime}\right) \gamma^{-1}=1 \text {, }
$$

and obtain

LEMMA 2. There exist polynomials $f$ and $h$ in $F(i)$ such that

$$
\left(f f^{\prime}-h h^{\prime}\right) \gamma=1 \text {. }
$$

Let now $r$ and $s$ be defined by

$$
r=\alpha f, s=\alpha h,
$$

where $\alpha$ is the quantity of (7) such that $z j=\alpha j^{2} z$ and $\alpha$ is in $F(i)$. Then $r$ and $s$ are in $F(i)$, and

$$
\left(r r^{\prime}-s s^{\prime}\right) \gamma\left(\alpha^{-1}\right)\left(\alpha^{-1}\right)^{\prime}=1 .
$$

But if $\delta=(j z)^{2}=j z j z=j \alpha j^{2} z z=\alpha_{\theta} g \gamma$, then $\delta_{\theta}=j(j z)^{2} j^{-1}=\left(j j z j^{-1}\right)^{2}=\left(\alpha^{-1} \alpha j^{2} z j^{-1}\right)^{2}$ $=\left(\alpha^{-1} z j j^{-1}\right)^{2}=\left(\alpha^{-1} z\right)^{2}=\left(\alpha^{-1}\right)\left(\alpha^{-1}\right)^{\prime} \gamma$, so that (18) gives

LEMMA 3. There exist quantities $r$ and $s$ in $F(i)$ such that, if

$$
\delta=(j z)^{2}=\alpha_{\theta} g \gamma,
$$

then

$$
\left(r r^{\prime}-s s^{\prime}\right) \delta_{\theta}=1
$$

If $a$ and $b$ are defined by

$$
a=s \alpha^{-1}, b=r_{\theta \theta},
$$

so that $b_{\theta}=r$, then

$$
Q \equiv\left[b_{\theta}\left(b_{\theta}\right)^{\prime}-a \alpha a^{\prime} \alpha^{\prime}\right], Q \delta_{\theta}=1
$$


3. The cyclic property. We shall now proceed to prove that $D$ is a cyclic algebra by the use of our fundamental existence theorem, Lemma 3. Consider the quantity

$$
X=a+b j+c j^{2},
$$

where we take $a$ and $b$ to be the polynomials of Lemma 3 which satisfy (22) and where $c$ will be chosen to be a polynomial in $i$ with coefficients in $F$. For every $c$ in $F(i)$, we have

$$
z X z^{-1} \equiv X^{\prime}=a^{\prime}+c^{\prime} \alpha \alpha_{\theta \theta} g j+b^{\prime} \alpha j^{2},
$$

so that

$$
X X^{\prime}=\left(a+b j+c j^{2}\right)\left(a^{\prime}+c^{\prime} \alpha \alpha_{\theta \theta} g j+b^{\prime} \cdot \alpha j^{2}\right)=A+B j+E j^{2},
$$

where $A$ is a polynomial in $i$ and

$$
\begin{aligned}
& B=a c^{\prime} \alpha \alpha_{\theta \theta} g+b\left(a^{\prime}\right)_{\theta}+c\left(b^{\prime} \alpha\right)_{\theta \theta} g=R c+S c^{\prime}+T, \\
& E=a b^{\prime} \alpha+b\left(c^{\prime} \alpha \alpha_{\theta \theta} z\right)_{\theta}+c\left(a^{\prime}\right)_{\theta \theta}=G c+H\left(c^{\prime}\right)_{\theta}+K .
\end{aligned}
$$

The quantities $B$ and $E$ are polynomials in $i$ and we have defined above

$$
\begin{aligned}
R \equiv\left(b^{\prime} \alpha\right)_{\theta \theta} g & =\left(b_{\theta}\right)^{\prime} \alpha_{\theta \theta} g, \quad S \equiv a \alpha \alpha_{\theta \theta} g, \quad T \equiv b\left(a^{\prime}\right)_{\theta}=b\left(a_{\theta \theta}\right)^{\prime}, \\
G & =\left(a^{\prime}\right)_{\theta \theta}=\left(a_{\theta}\right)^{\prime}, \quad H=b \alpha_{\theta} \alpha g, \quad K=a b^{\prime} \alpha,
\end{aligned}
$$

all in $F(i)$. Now

$$
\left(g^{\prime}\right)_{\theta}=\left(g_{\theta \theta}\right)^{\prime}=g^{\prime}, \quad\left(g^{\prime}\right)_{\theta \theta}=\left[\left(g^{\prime}\right)_{\theta}\right]_{\theta}=\left(g^{\prime}\right)_{\theta}=g .
$$

Transforming $B$ by $z$ we have

$$
B^{\prime}=R^{\prime} c^{\prime}+S^{\prime} c+T^{\prime}
$$

whence

$$
\begin{aligned}
R^{\prime} B-S B^{\prime} & =R^{\prime} R c+R^{\prime} S c^{\prime}+R^{\prime} T-S R^{\prime} c^{\prime}-S S^{\prime} c-S T^{\prime} \\
& =\left(R R^{\prime}-S S^{\prime}\right) c-\left(S T^{\prime}-R^{\prime} T\right) .
\end{aligned}
$$

But

$$
\begin{aligned}
R R^{\prime}-S S^{\prime} & =\left(b_{\theta}\right)^{\prime} \alpha_{\theta \theta} g b_{\theta}\left(\alpha_{\theta \theta}\right)^{\prime} g^{\prime}-a \alpha \alpha_{\theta \theta} g a^{\prime} \alpha^{\prime}\left(\alpha_{\theta \theta}\right)^{\prime} g^{\prime} \\
& =g g^{\prime} \alpha_{\theta \theta}\left(\alpha_{\theta \theta}\right)^{\prime}\left[b_{\theta}\left(b_{\theta}\right)^{\prime}-a \alpha a^{\prime} \alpha^{\prime}\right]=g g^{\prime} \alpha_{\theta \theta}\left(\alpha_{\theta \theta}\right)^{\prime} Q .
\end{aligned}
$$

From (19) $\delta_{\theta}=\alpha_{\theta \theta} g \gamma_{\theta}$, so that, utilizing the relation $Q \delta_{\theta}=1$, we have

$$
R R^{\prime}-S S^{\prime}=g g^{\prime} \alpha_{\theta \theta}\left(\alpha_{\theta \theta}\right)^{\prime} \delta_{\theta}^{-1}=g^{\prime}\left(\alpha_{\theta \theta}\right)^{\prime}\left(\gamma_{\theta}\right)^{-1} \neq 0,
$$

since $g, \alpha$ and $\gamma$ are all not zero in a division algebra. Hence $R R^{\prime}-S S^{\prime}$ has an inverse $\left(R R^{\prime}-S S^{\prime}\right)^{-1}$ in $F(i)$, and if we define the quantity $c$ by 


$$
c=\left(S T^{\prime}-R^{\prime} T\right)\left(R R^{\prime}-S S^{\prime}\right)^{-1}
$$

then

$$
R^{\prime} B-S B^{\prime}=\left(S T^{\prime}-R^{\prime} T\right)-\left(S T^{\prime}-R^{\prime} T\right)=0 .
$$

We shall henceforth consider the quantity $X$ as completely defined in (23) with the $a$ and $b$ of Lemma 3 and the $c$ of (35), so that (36) is satisfied. Transforming (36) by $z$ we have

$$
R B^{\prime}-B S^{\prime}=0
$$

whence

$$
R\left(R^{\prime} B-S B^{\prime}\right)+S\left(-B S^{\prime}+R B^{\prime}\right)=B\left(R R^{\prime}-S S^{\prime}\right)=0 .
$$

But $R R^{\prime}-S S^{\prime}$ has an inverse in $F(i)$, whence $B=0$.

We consider now the polynomial $E$. We first compute

$$
S T^{\prime}-R^{\prime} T=a \alpha \alpha_{\theta \theta} g b^{\prime} a_{\theta \theta}-b_{\theta}\left(\alpha_{\theta \theta}\right)^{\prime} g^{\prime} b\left(a_{\theta \theta}\right)^{\prime} .
$$

Next

$$
\begin{aligned}
H\left(K^{\prime}\right)_{\theta}-\left(G^{\prime}\right)_{\theta} K & =b \alpha_{\theta} \alpha g\left(a^{\prime}\right)_{\theta} b_{\theta}\left(\alpha^{\prime}\right)_{\theta}-a_{\theta \theta} a b^{\prime} \alpha \\
& =-\left[\alpha a a_{\theta \theta} b^{\prime}-\alpha \alpha_{\theta} g b b_{\theta}\left(\alpha_{\theta \theta}\right)^{\prime}\left(a_{\theta \theta}\right)^{\prime}\right]
\end{aligned}
$$

so that

$$
-\alpha_{\theta \theta} g\left[H\left(K^{\prime}\right)_{\theta}-\left(G^{\prime}\right)_{\theta} K\right]=a \alpha a_{\theta \theta} \alpha_{\theta \theta} b^{\prime} g-b b_{\theta}\left(\alpha \alpha_{\theta} \alpha_{\theta \theta} g^{2}\right)\left(a_{\theta \theta}\right)^{\prime}\left(\alpha_{\theta \theta}\right)^{\prime} .
$$

But $j^{3}=g, g^{\prime}=z g z^{-1}=z j^{3} z^{-1}=\left(z j z^{-1}\right)^{3}=\left(\alpha j^{2} z z^{-1}\right)^{3}=\left(\alpha j^{2}\right)^{3}=\alpha j^{2} \alpha j^{2} \alpha j^{2}=\alpha \alpha_{\theta \theta} \alpha{ }_{\theta} g^{2}$, and we have the relations

$$
\begin{aligned}
g^{\prime} & =\alpha \alpha_{\theta} \alpha_{\theta \theta} g^{2}, \\
g & =\alpha^{\prime}\left(\alpha_{\theta \theta}\right)^{\prime}\left(\alpha_{\theta}\right)^{\prime}\left(g^{\prime}\right)^{2}=\alpha^{\prime}\left(\alpha^{\prime}\right)_{\theta}\left(\alpha^{\prime}\right)_{\theta \theta}\left(g^{\prime}\right)^{2} .
\end{aligned}
$$

Substituting (42) in (41) and comparing with (39) we write immediately

$$
S T^{\prime}-R^{\prime} T=-\alpha_{\theta \theta} g\left[H\left(K^{\prime}\right)_{\theta}-\left(G^{\prime}\right)_{\theta} K\right] \text {. }
$$

We also have, by the use of $(11),\left[\left(\alpha_{\theta}\right)^{\prime}\right]_{\theta}=\left[\left(\alpha^{\prime}\right)_{\theta \theta}\right]_{\theta}=\alpha^{\prime}$, and

$$
G\left(G^{\prime}\right)_{\theta}-H\left(H^{\prime}\right)_{\theta}=\left(a_{\theta}\right)^{\prime} a_{\theta \theta}-b \alpha \alpha_{\theta} g\left(b^{\prime}\right)_{\theta}\left(\alpha^{\prime}\right)_{\theta} \alpha^{\prime} g^{\prime} .
$$

But then

$$
\begin{aligned}
-g \alpha_{\theta \theta}\left(\alpha^{\prime}\right)_{\theta \theta}\left[G\left(G^{\prime}\right)_{\theta}\right. & \left.-H\left(H^{\prime}\right)_{\theta}\right] \\
& =\left(\alpha \alpha_{\theta} \alpha_{\theta \theta} g^{2}\right)\left[\alpha^{\prime}\left(\alpha^{\prime}\right)_{\theta}\left(\alpha^{\prime}\right)_{\theta \theta} g^{\prime}\right] b\left(b^{\prime}\right)_{\theta}-g a_{\theta \theta}\left(a^{\prime}\right)_{\theta \theta} \alpha_{\theta \theta}\left(\alpha^{\prime}\right)_{\theta \theta}
\end{aligned}
$$

which by (42) and (43) has the value

$$
g\left[b\left(b^{\prime}\right)_{\theta}-a_{\theta \theta} \alpha_{\theta \theta}\left(a^{\prime}\right)_{\theta \theta}\left(\alpha^{\prime}\right)_{\theta \theta}\right] .
$$


But if

$$
Q=b_{\theta}\left(b_{\theta}\right)^{\prime}-a \alpha a^{\prime} \alpha^{\prime}=b_{\theta}\left(b^{\prime}\right)_{\theta \theta}-a \alpha a^{\prime} \alpha^{\prime},
$$

ther from (22), $\delta_{\theta} Q=1, Q \neq 0$,

$$
Q_{\theta \theta}=b\left(b^{\prime}\right)_{\theta}-a_{\theta \theta} \alpha_{\theta \theta}\left(a^{\prime}\right)_{\theta \theta}\left(\alpha^{\prime}\right)_{\theta \theta} .
$$

Hence

$$
-\alpha_{\theta \theta}\left(\alpha^{\prime}\right)_{\theta \theta}\left[G\left(G^{\prime}\right)_{\theta}-H\left(H^{\prime}\right)_{\theta}\right]=g Q_{\theta \theta} \neq 0,
$$

so that

(51) $\frac{H\left(K^{\prime}\right)_{\theta}-\left(G^{\prime}\right)_{\theta} K}{G\left(G^{\prime}\right)_{\theta}-H\left(H^{\prime}\right)_{\theta}}=\frac{-\alpha_{\theta \theta} g\left[H\left(K^{\prime}\right)_{\theta}-\left(G^{\prime}\right)_{\theta} K\right]}{-\alpha_{\theta \theta} g\left[G\left(G^{\prime}\right)_{\theta}-H\left(H^{\prime}\right)_{\theta}\right]} \cdot \frac{\left(\alpha^{\prime}\right)_{\theta \theta}}{\left(\alpha^{\prime}\right)_{\theta \theta}}=\frac{\left(\alpha^{\prime}\right)_{\theta \theta}\left[S T^{\prime}-R^{\prime} T\right]}{g Q_{\theta \theta}}$.

From (22) we have $\delta_{\theta} Q=1$, so that

$$
j^{2}\left(\delta_{\theta} Q\right) j^{-2}=1=\delta Q_{\theta \theta}=\delta_{\theta} Q,
$$

whence

$$
\frac{\delta_{\theta} Q}{\gamma_{\theta}}=\frac{\delta Q_{\theta \theta}}{\gamma_{\theta}}
$$

Now $\delta=\gamma \alpha_{\theta} g$ from (19), while $\delta=(j z)^{2}$ is commutative with $j z$ and equals its transform by $j z$, the quantity $\left(\delta^{\prime}\right)_{\theta}$. Hence

$$
g=g_{\theta}, \quad \alpha_{\theta} g=\frac{\delta}{\gamma}, \quad \alpha_{\theta \theta} g=\frac{\delta_{\theta}}{\gamma_{\theta}}, \alpha g=\frac{\delta_{\theta \theta}}{\gamma_{\theta \theta}},
$$

and

$$
\alpha^{\prime} g^{\prime}=\frac{\left(\delta_{\theta \theta}\right)^{\prime}}{\left(\gamma_{\theta \theta}\right)^{\prime}}=\frac{\left(\delta^{\prime}\right)_{\theta}}{\left(\gamma^{\prime}\right)_{\theta}}=\frac{\delta}{\gamma_{\theta}}
$$

since $\gamma=\gamma^{\prime}$. Equation (53) becomes

$$
\alpha_{\theta \theta} g Q=\alpha^{\prime} g^{\prime} Q_{\theta \theta} .
$$

From (43) $g=\alpha^{\prime}\left(\alpha^{\prime}\right)_{\theta}\left(\alpha^{\prime}\right)_{\theta \theta}\left(g^{\prime}\right)^{2}$, so that

$$
\alpha^{\prime} g^{\prime} Q_{\theta \theta}=\left(\alpha^{\prime} g^{\prime}\right)\left[\left(\alpha^{\prime}\right)_{\theta}\left(\alpha^{\prime}\right)_{\theta \theta} g^{\prime}\right] \alpha_{\nu \theta} Q
$$

and

$$
Q_{\theta \theta}=\left(\alpha^{\prime}\right)_{\theta \theta}\left(\alpha_{\theta \theta}\right)^{\prime} \alpha_{\theta \theta} g^{\prime} Q
$$

It follows now that

$$
g Q_{\theta \theta} /\left(\alpha^{\prime}\right)_{\theta \theta}=g g^{\prime} \alpha_{\theta \theta}\left(\alpha_{\theta \theta}\right)^{\prime} Q=R R^{\prime}-S S^{\prime},
$$


by (33). Using (35) and (51) we have

$$
c=\left(S T^{\prime}-R^{\prime} T\right)\left(R R^{\prime}-S S^{\prime}\right)^{-1}=\left[H\left(K^{\prime}\right)_{\theta}-\left(G^{\prime}\right)_{\theta} K\right]\left[G\left(G^{\prime}\right)_{\theta}-H\left(H^{\prime}\right)_{\theta}\right]^{-1} .
$$

We have now demonstrated that

$$
\left[G\left(G^{\prime}\right)_{\theta}-H\left(H^{\prime}\right)_{\theta}\right] c-\left[H\left(K^{\prime}\right)_{\theta}-\left(G^{\prime}\right)_{\theta} K\right]=0,
$$

a relation very similar to (36). In fact, since $E$ is given by $(27)$, and $\left[\left(c^{\prime}\right)_{\theta}\right]^{\prime}$ $=c_{\theta \theta},\left(c_{\theta \theta}\right)_{\theta}=c$,

$$
\left(E^{\prime}\right)_{\theta}=\left(G^{\prime}\right)_{\theta}\left(c^{\prime}\right)_{\theta}+\left(H^{\prime}\right)_{\theta} C+\left(K^{\prime}\right)_{\theta}
$$

so that, by (60),

$$
\begin{aligned}
\left(G^{\prime}\right)_{\theta} E-H\left(E^{\prime}\right)_{\theta}= & \left(G^{\prime}\right)_{\theta} G c+\left(G^{\prime}\right)_{\theta} H\left(c^{\prime}\right)_{\theta}+\left(G^{\prime}\right)_{\theta} K \\
& -H\left(G^{\prime}\right)_{\theta}\left(c^{\prime}\right)_{\theta}-H\left(H^{\prime}\right)_{\theta} c-H\left(K^{\prime}\right)_{\theta} \\
= & {\left.\left[G^{\prime} G^{\prime}\right)_{\theta}-H\left(H^{\prime}\right)_{\theta}\right] c-\left[H\left(K^{\prime}\right)_{\theta}-\left(G^{\prime}\right)_{\theta} K\right]=0 . }
\end{aligned}
$$

Transforming (62) by $j z$ we have

$$
G\left(E^{\prime}\right)_{\theta}-\left(H^{\prime}\right)_{\theta} E=0,
$$

and

(64) $0=G\left[\left(G^{\prime}\right)_{\theta} E-H\left(E^{\prime}\right)_{\theta}\right]+H\left[G\left(E^{\prime}\right)_{\theta}-\left(H^{\prime}\right)_{\theta} E\right]=\left[G\left(G^{\prime}\right)_{\theta}-H\left(H^{\prime}\right)_{\theta}\right] E=0$.

It follows from (50) that $E=0$ and that (25) becomes

$$
X X^{\prime}=A \text { in } F(i) \text {. }
$$

But then

$$
(X z)^{2}=X z X z=X X^{\prime} \gamma=A \gamma=t \text { in } F(i),
$$

since $\gamma$ is in $F(i)$, and $X^{\prime}$ was defined so that $z X=X^{\prime} z$ in (24).

Let first $b$ and $a$ be both not zero, so that since $X z$ is commutative with its square,

$$
t X z=\left(t a+t b j+t c j^{2}\right) z=X z t=\left(a+b j+c j^{2}\right) t^{\prime} z .
$$

Hence

$$
t a+t b j+t c j^{2}=t^{\prime} a+\left(t^{\prime}\right)_{\theta} b j+\left(t^{\prime}\right)_{\theta \theta} c j^{2},
$$

and since (6) are a basis of $D, t a=t^{\prime} a, t b=\left(t^{\prime}\right)_{\theta} b$. Since $a$ is not zero, $t=t^{\prime}$ is in $F\left(i^{2}\right)$. Since also $b$ is not zero, $t=\left(t^{\prime}\right)_{\theta}=t_{\theta}$ is in $F$. It follows that when $a b \neq 0$ we have shown that there exists a quantity $X$ in the algebra

$$
\Sigma=\left(i^{s} j^{r}\right) \quad(s=0,1, \cdots, 5 ; r=0,1,2),
$$


such that $X \neq 0$ and

$$
(X z)^{2}=\lambda \text { in } F .
$$

Suppose next that $a$ were zero so that from its origin (21) we have $s=0$ and $h=0$ in (17). Then (16) becomes

$$
f f^{\prime} \gamma=(f z)^{2}=1,
$$

while then obviously $f$ cannot be zero and $f=\alpha^{-1} r=\alpha^{-1} b_{\theta}$ is in $F(i)$ and in $\Sigma$. Again we have (67) for $X \neq 0$ in $\Sigma$. Finally the only remaining case is $b$ zero. Then $r_{\theta \theta}=0$ so that $r=0$ in (17), and hence the quantity $f$ is zero. Equation (16) now becomes $h h^{\prime} \gamma=(h z)^{2}=-1$, and since then $h=\alpha^{-1} s=a$ in $F(i)$ cannot be zero when $(h z)^{2}=-1$, we have again proved the existence of $X \neq 0$ in $\Sigma$ and satisfying (67). Hence in all cases we have

LEMMA 4. There exists a quantity $X$ in $\Sigma$ such that $X \neq 0$, and if $y=X z$ then

$$
y^{2}=\lambda \text { in } F \text {. }
$$

The quantities $1, v, y, v y$ are linearly independent with respect to $F$, for otherwise (6) could not be a basis of $D$ when $X \neq 0$. A relation of the form

$$
\xi_{1}+\xi_{2} v+\left(\xi_{3}+\xi_{4} v\right) X_{z}=0,
$$

with $\xi_{1}, \xi_{2}, \xi_{3}, \xi_{4}$ not all zero and in $F$, would then evidently express $z$ as a quantity of $\Sigma$. Also $v^{2}=\rho, y^{2}=\lambda, y v=X z v=-X v z=-v X z=-v y$, since $v$, a polynomial in $i$ commutative with $j$, is commutative with $X$. But the linear set

$$
\Gamma=(1, v ; y, v y)
$$

is evidently a generalized quaternion algebra over $F$, and is a normal division cyclic algebra over $F$. Hence $D$, containing $\Gamma$, is the direct product* of $\Gamma$ and another algebra $\Omega$ of order nine over $F$. Since $D$ is a normal algebra, so is necessarily $\Omega, \dagger$ so that $\Omega$ is a cyclic algebra $\ddagger$ of order nine. Hence $D$, the direct product of algebra $\Gamma$ and algebra $\Omega$, is a cyclic algebra. $\dagger$

THEOREM. Every normal division algebra of type $R$ in thirty-six units is a cyclic algebra.

* A theorem of Wedderburn; cf. Algebras and their Arithmetics, p. 237.

$\dagger$ For the first and second of the above references respectively see Theorems 7 and 16 of the author's paper, On direct products, cyclic division algebras, and pure Riemann matrices, which appears in the present number of these Transactions.

$\ddagger$ A theorem of Wedderburn, these Transactions, vol. 22 (1921), pp. 129-135.

Columbia University, New York, N. Y. 